\title{
Quantitative trait locus analysis for kernel width using maize recombinant inbred lines
}

\author{
G.Q. Hui ${ }^{1}$, G.Q. Wen ${ }^{2}$, X.H. Liu², H.P. Yang ${ }^{1}$, Q. Luo', H.X. Song ${ }^{3}$, L. Wen ${ }^{3}$, \\ Y. Sun ${ }^{4}$ and H.M. Zhang ${ }^{1,5}$ \\ ${ }^{1}$ Maize Research Institute, Shanxi Academy of Agricultural Sciences, \\ Xinzhou, China \\ ${ }^{2}$ Key Laboratory of Southwest China Wildlife Resources Conservation \\ (Ministry of Education), College of Life Science, China West Normal University, \\ Nanchong, China \\ ${ }^{3}$ Biological Engineering College, Shanxi University, Taiyuan, China \\ ${ }^{4}$ Biotechnology Research Center, Shanxi Academy of Agricultural Sciences, \\ Taiyuan, China \\ ${ }^{5}$ Key Laboratory of Crop Gene Resources and Germplasm Enhancement on Loess \\ Plateau, Ministry of Agriculture, Taiyuan, China \\ Corresponding author: X.H. Liu \\ E-mail: 350783409@qq.com
}

Genet. Mol. Res. 14 (4): 14496-14502 (2015)

Received June 5, 2015

Accepted September 9, 2015

Published November 18, 2015

DOI http://dx.doi.org/10.4238/2015.November.18.12

ABSTRACT. Maize (Zea mays L.) kernel width is one of the most important traits that is related to yield and appearance. To understand its genetic mechanisms more clearly, a recombinant inbred line (RIL) segregation population consisting of 239 RILs was used for quantitative trait locus (QTL) mapping for kernel width. We found four QTLs on chromosomes 3 (one), 5 (two), and 10 (one). The QTLs were close to their adjacent markers, with a range of $0-23.8 \mathrm{cM}$, and explained $6.2-19.7 \%$ of the phenotypic variation. The three QTLs on chromosomes 3 and 5 had positive additive effects, and to a certain extent increased kernel width, whereas the one on chromosome 10 exhibited negative additive effects and decreased kernel 
width. These results can be used for gene cloning and marker-assisted selection in maize-breeding programs.

Key words: Maize (Zea mays L.); Kernel width; Quantitative trait locus; Genetic mechanism

\section{INTRODUCTION}

Maize (Zea mays L.) is originally from the Americas, and has been cultivated for thousands of years. It has many merits, including its adaptability, high yield, and excellent quality, and has become the second most important crop after wheat. With the reduction in arable land and the increasing use of land for city construction, maintaining and increasing maize yields is a very important long-term task, and research on the genetic mechanisms of maize has already become a key aim for breeders.

Quantitative trait locus (QTL) mapping is frequently and effectively used for investigating genetic mechanisms in crops, and is also used in the important preliminary work for marker-assisted selection (MAS) and gene cloning. Maize kernel structure is a crucial trait that defines yield and appearance, but studies on the genetic mechanisms of kernel structure are few compared to those on other agronomic traits, such as yield (Huang et al., 2010; Peng et al., 2013; Xu et al., 2014), plant morphology (Chen et al., 2014; Yu et al., 2014), disease resistance (Tao et al., 2013; Zambrano et al., 2014; Xu et al., 2014), and drought tolerance (Rahman et al., 2011; Almeida et al., 2013). However, a few studies have conducted QTL mapping for maize kernel width and investigated correlations with kernel structure. Li et al. (2009) mapped one QTL on chromosome 3, and Peng et al. (2013) identified three QTLs on chromosomes 3, 7, and 10. Recently, Zhang et al. (2014) detected six QTLs, and Liu et al. (2014) identified up to 16 . The use of different parental lines, population types, genetic mapping, and mapping methods probably led to the different results obtained, including QTL number, chromosomal location, and genetic effects. Therefore, selecting new parental materials and segregation population is necessary and significant in the study on QTL mapping for maize kernel width.

In this study, a recombinant inbred line (RIL) population derived from a cross between Mo17 (a representative inbred line of the Lancaster heterosis group from the USA) and Huangzaosi (a representative inbred line of the Tansipingtou heterosis group from China) was used for the QTL mapping of kernel width, and the aims were to 1) ascertain its genetic mechanisms, 2) identify QTLs that could be mapped, and 3) identify molecular markers that could be used in maize MAS.

\section{MATERIAL AND METHODS}

\section{Field experiments and statistical analysis}

A total of 241 lines, which consisted of 239 RILs and the two parental lines (Mo17 and Huangzaosi), were planted at the Nanchong Agricultural Research Academy, Nanchong City, China. After harvesting, the kernel widths of 20 randomly selected kernels from each line were measured using an electronic digital caliper $(\mathrm{mm})$, and their mean values were calculated using Microsoft Excel 2010. Descriptive statistics, including ranges, minima, maxima, means, standard deviations, skewness, kurtosis, and coefficients of variation, were obtained from the RILs using SPSS version 11.5 (www.spss.com). In addition, a frequency distribution was calculated using the same software, based on the trait values of the RILs. 


\section{QTL mapping for kernel width}

Based upon the previous linkage map that included 100 microsatellite markers and covered $1421.5 \mathrm{cM}$ of the genome (Liu et al., 2009), QTL scanning was performed by composite interval mapping in Windows QTL Cartographer version 2.5 (Wang et al., 2012), with a 10-cM widow size, model 6 (standard model), 5 control markers, and a backward regression method. The log 10 of the odds ratio (LOD) threshold value was determined by a 1000-permutation test $(\alpha=0.05)$ (Churchill and Doerge, 1994; Doerge and Churchill, 1996). The chromosomal positions, additive genetic effects, and percentages of phenotypic variation of the detected QTLs were estimated at the peak of the LOD curve over the threshold values. The identified QTLs were mapped onto a marker linkage map using the MapChart 2.1 software (Voorrips, 2002).

\section{RESULTS}

\section{Phenotypic observations}

Mo17 had larger kernel widths than Huangzaosi (Table 1), and kernel widths in the RILs varied between 6.69 and $9.70 \mathrm{~mm}$ (Table 2). The values followed a normal distribution (Figure 1), confirming that kernel width is a quantitative trait and is controlled by several genes.

\section{Table 1. Mean kernel width values of parental inbred lines.}

\begin{tabular}{lc}
\hline Parental line & Kernel width $(\mathrm{mm})$ \\
\hline Mo17 & 9.60 \\
Huangzaosi & 8.59 \\
\hline
\end{tabular}

Table 2. Descriptive statistics of kernel width in maize recombinant inbred lines.

\begin{tabular}{lccccccc}
\hline Range & Minimum & Maximum & Mean & SD & Skewness & Kurtosis & CV (\%) \\
\hline 3.01 & 6.69 & 9.70 & 8.23 & 0.58 & -0.034 & -0.389 & 7.05 \\
\hline
\end{tabular}

SD, standard deviation; CV, coefficient of variation.

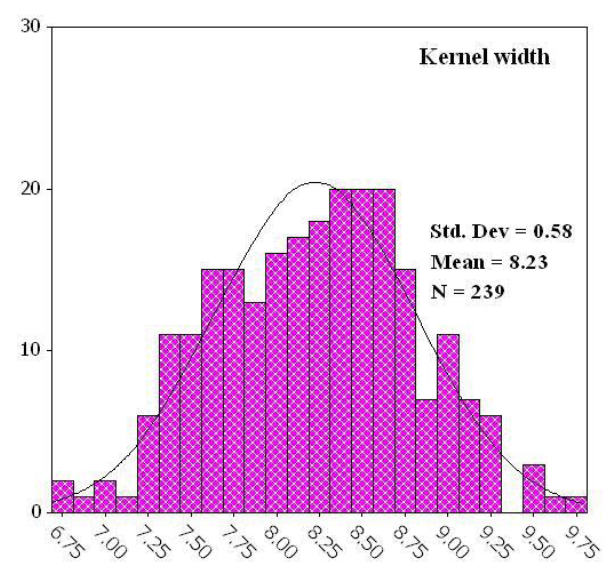

Figure 1. Frequency distribution of kernel widths in maize recombinant inbred lines. 


\section{QTL mapping}

The permutation test indicated that the LOD threshold value should be set at 2.53 , and QTL scanning was performed based on this value (Figure 2). One, two, and one QTLs were found on chromosomes 3,5 , and 10 , respectively, and all exhibited positive additive effects except for the one on chromosome 10.

The chromosomal distributions of the four QTLs are shown in Figure 3, and their marker intervals were Bnlg1108-Umc2048 (Qt/3), Bnlg1106-Dupssr10 (Qt/5-1), Dupssr10-Umc1966 (Qt/52), and Phi050-Umc1196 (Qt/10). The genetic distances between the QTLs and their closest markers ranged from 0 to $23.8 \mathrm{cM}$, and the QTL Qt/5-2 on chromosome 5 was $0 \mathrm{cM}$ to its closest marker, suggesting that it may have been co-inherited with the Dupssr10 marker.

The genetic parameters of the four QTLs are listed in Table 3. All of the LOD values were over 3.5. The three QTLs on chromosomes 3 and 5 (Qt/3, Qt/5-1, and Qt/5-2) exhibited positive additive effects and increased kernel width by $0.25,0.36$, and $0.15 \mathrm{~mm}$, respectively, and explained $60.7 \%$ of the total phenotypic variation. However, the QTL on chromosome 10 (Qt/10) exhibited negative additive effects, and decreased kernel width by up to $0.26 \mathrm{~mm}$; according to its $R^{2}$ value it explained $19.7 \%$ of the total phenotypic variation.

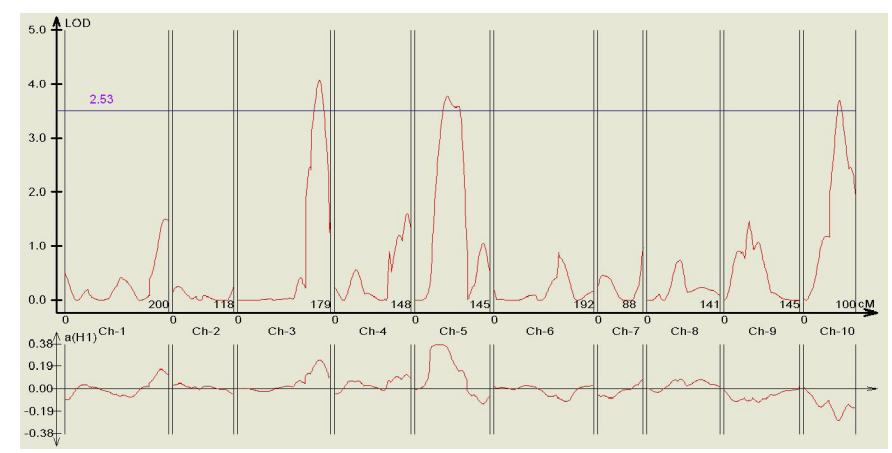

Figure 2. Composite interval mapping in quantitative trait locus analysis for kernel width.

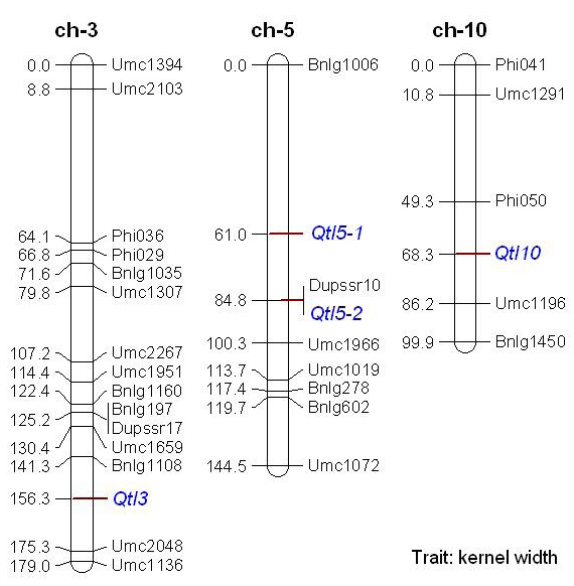

Figure 3. Chromosomal distribution of quantitative trait loci for maize kernel width. 
Table 3. Quantitative trait loci for kernel width and their genetic parameters.

\begin{tabular}{lrlcrrr}
\hline QTL & Chr. & Adjacent marker & Position (cM) & LOD & Additive effect & R2 (\%) \\
\hline Qt/3 & 3 & Bnlg1108 & 156.3 & 4.1 & 0.25 & 17.2 \\
Qt/5-1 & 5 & Dupssr10 & 61.0 & 3.8 & 0.36 \\
Qt/5-2 & 5 & Dupssr10 & 84.8 & 3.6 & 0.15 & 37.3 \\
Qt/10 & 10 & Umc1196 & 68.3 & 3.7 & -0.26 & 19.7 \\
\hline
\end{tabular}

QTL, quantitative trait locus; Chr., chromosome number; LOD, $\log 10$ of odds ratio; R2, percentage of phenotypic variance explained by QTL.

\section{DISCUSSION}

Four QTLs were identified on chromosomes 3 (one), 5 (two), and 10 (one). The three on chromosomes 3 and 5 exhibited positive additive effects, whereas the one on chromosome 10 exhibited negative additive effects.

Our results differ from those of previous studies in many aspects, due to different parental materials, segregation populations, marker maps, or ecological environments (Table 4).

Table 4. Quantitative trait loci for maize kernel width reported in different studies.

\begin{tabular}{|c|c|c|c|c|c|}
\hline Reference & Parents & Chr. & QTL & Marker interval & Additive effect \\
\hline Li et al., 2009 & Qi319 x Huangzaosi & 8 & No name & Umc1139-Umc1157 & - \\
\hline \multirow[t]{2}{*}{ Peng et al., 2013} & \multirow[t]{2}{*}{ Qi319 x Huangzaosi } & 3 & Qqkwid3 & Bnlg1496-umc1010 & - \\
\hline & & 10 & Qqkwid10 & Bnlg1677-Umc2172 & + \\
\hline Peng et al., 2013 & Ye478 x Huangzaosi & 7 & Yqkwid7 & Umc1016-Bnlg1094 & + \\
\hline \multirow[t]{6}{*}{ Zhang et al., 2014} & \multirow[t]{6}{*}{ Xu178 x HuangC } & 2 & $q K W I 2 a$ & Umc1497-Umc2380 & - \\
\hline & & 2 & $q K W I 2 b$ & Umc1185-Umc1579 & + \\
\hline & & 6 & $q K W I 6 a$ & Umc1341-Umc1912 & + \\
\hline & & 6 & $q K W I 6 b$ & Umc1912-Phi452693 & + \\
\hline & & 6 & $q K W I 6 c$ & Umc1444-Bnlg249 & + \\
\hline & & 7 & qKWI7 & Bnlg1305-Dupssr11 & + \\
\hline \multirow[t]{16}{*}{ Liu et al., 2014} & \multirow[t]{16}{*}{ V67 x Mc } & 1 & $q K W 1-1$ & umc2225-bnlg1007 & - \\
\hline & & 1 & $q K W 1-2$ & bnlg1007-bnlg439 & - \\
\hline & & 2 & $q K W 2-1$ & umc2245-umc1227 & + \\
\hline & & 2 & $q K W 2-2$ & bnlg1831-bnlg1138 & + \\
\hline & & 2 & $q K W 2-3$ & umc2023-umc1890 & + \\
\hline & & 2 & $q K W 2-4$ & umc1946-umc2625 & + \\
\hline & & 2 & $q K W 2-5$ & bnlg1662-bnlg1316 & + \\
\hline & & 2 & $q K W 2-6$ & bnlg1316-umc1464 & + \\
\hline & & 2 & $q K W 2-7$ & umc1526-umc1230 & + \\
\hline & & 3 & $q K W 3$ & umc1320-umc1273 & + \\
\hline & & 4 & $q K W 4-1$ & umc1667-umc2041 & - \\
\hline & & 4 & $q K W 4-2$ & umc1051-bnlg292b & - \\
\hline & & 5 & $q K W 5$ & umc2294-umc2161 & + \\
\hline & & 6 & $q K W 6$ & phi070-umc2165 & + \\
\hline & & 9 & $q K W 9-1$ & umc2084-bnlg1583 & - \\
\hline & & 9 & $q K W 9-2$ & umc2346-umc1714 & - \\
\hline \multirow[t]{4}{*}{ This study } & \multirow[t]{4}{*}{ Mo17 x Huangzaosi } & 3 & Qt/3 & Bnlg1108-Umc2048 & + \\
\hline & & 5 & Qt/5-1 & Bnlg1006-Dupssr10 & + \\
\hline & & 5 & $Q t / 5-2$ & Dupssr10-Umc1966 & + \\
\hline & & 10 & Qt/10 & Phi050-Umc1196 & - \\
\hline
\end{tabular}

Chr., chromosome number; QTL, quantitative trait locus.

On chromosome 3, Peng et al. (2013) identified one QTL for kernel width, but it was different to the QTL located on the same chromosome in our study, due to opposite additive effects. Liu et al. (2014) mapped one QTL on chromosome 3 that exhibited positive additive effects, as we found in the present study, with the addition of the same chromosomal bin region; therefore, 
the two QTLs are probably the same loci and are associated with kernel width. For chromosome 10, Peng et al. (2013) mapped one QTL that exhibited positive additive effects, whereas the QTL on the same chromosome in our study exhibited the opposite genetic effects, so the two QTLs are different. For chromosome 5, Liu et al. (2014) identified one QTL possessing positive additive effects, while in our stuy, two QTLs were detected, both of them had positive additive effects.

The three QTLs (Qt/5-1, Qt/10, and Qt/3) had large genetic distances (up to $10.0 \mathrm{cM}$ ) to their respective closest markers. Qt/5-1 on chromosome 5 had the maximum value (over $20.0 \mathrm{cM}$ ), and was followed in descending order by Qt/10 (17.9 cM) and Qt/3 (15.0 cM) on chromosomes 10 and 3, respectively. Therefore, these three QTLs need to be more finely mapped; this could be accomplished by adding molecular markers to the corresponding chromosomal regions. However, because the genetic distance between Qt/5-2 and the Dupssr10 marker (bin5.04) was $0 \mathrm{cM}$, they were probably co-inherited; this suggests that this gene could be cloned or be included in maize MAS.

\section{Conflicts of interest}

The authors declare no conflict of interest.

\section{ACKNOWLEDGMENTS}

Research supported by the Scientific Research Fund of Sichuan Provincial Education Department of China (\#13ZA0012) and the National Twelfth Five-Year Plan for Science \& Technology Support of China (\#2011BAD35B01).

\section{REFERENCES}

Almeida GD, Makumbi D, Magorokosho C, Nair S, et al. (2013). QTL mapping in three tropical maize populations reveals a set of constitutive and adaptive genomic regions for drought tolerance. Theor. Appl. Genet. 126: 583-600.

Chen Z, Wang B, Dong X, Liu H, et al. (2014). An ultra-high density bin-map for rapid QTL mapping for tassel and ear architecture in a large $F_{2}$ maize population. BMC Genomics 15: e433.

Churchill GA and Doerge RW (1994). Empirical threshold values for quantitative trait mapping. Genetics 138: 963-971.

Doerge RW and Churchill GA (1996). Permutation tests for multiple loci affecting a quantitative character. Genetics 142: 285-294.

Huang YF, Madur D, Combes V, Ky CL, et al. (2010). The genetic architecture of grain yield and related traits in Zea maize L. revealed by comparing intermated and conventional populations. Genetics 186: 395-404.

Li YX, Wang Y, Shi YS, Song YC, et al. (2009). Correlation analysis and QTL mapping for traits of kernel structure and yield components in maize. Sci. Agric. Sin. 42: 408-418.

Liu XH, Tan ZB and Rong TZ (2009). Molecular mapping of a major QTL conferring resistance to SCMV based on immortal RIL population in maize. Euphytica 167: 229-235.

Liu Y, Wang L, Sun C, Zhang Z, et al. (2014). Genetic analysis and major QTL detection for maize kernel size and weight in multi-environments. Theor. Appl. Genet. 127: 1019-1037.

Peng B, Li Y, Wang Y, Liu C, et al. (2013). Correlations and comparisons of quantitative trait loci with family per se and testcross performance for grain yield and related traits in maize. Theor. Appl. Genet. 126: 773-789.

Rahman H, Pekic S, Lazic-Jancic V, Quarrie SA, et al. (2011). Molecular mapping of quantitative trait loci for drought tolerance in maize plants. Genet. Mol. Res. 10: 889-901.

Tao Y, Liu Q, Wang H, Zhang Y, et al. (2013). Identification and fine-mapping of a QTL, qMrdd1, that confers recessive resistance to maize rough dwarf disease. BMC Plant Biol. 13: e145.

Voorrips RE (2002). MapChart: software for the graphical presentation of linkage maps and QTLs. J. Hered. 93: 77-78.

Wang S, Basten CJ and Zeng Z-B (2012). Windows QTL Cartographer 2.5. Department of Statistics, North Carolina State University, Raleigh, NC, USA. Available at [http://statgen.ncsu.edu/qtlcart/WQTLCart.htm]. Accessed March 10, 2010.

Xu L, Zhang Y, Shao S, Chen W, et al. (2014). High-resolution mapping and characterization of qRgls2, a major quantitative trait locus involved in maize resistance to gray leaf spot. BMC Plant Biol. 14: e230. 
Yu YT, Li GK, Yang ZL, Hu JG, et al. (2014). Identification of a major quantitative trait locus for ear size induced by space flight in sweet corn. Genet. Mol. Res. 13: 3069-3078.

Zambrano JL, Jones MW, Brenner E, Francis DM, et al. (2014). Genetic analysis of resistance to six virus diseases in a multiple virus-resistant maize inbred line. Theor. Appl. Genet. 127: 867-880.

Zhang Z, Liu Z, Hu Y, Li W, et al. (2014). QTL analysis of kernel-related traits in maize using an immortalized F2 Population. PLoS One 9: e89645. 